\title{
ATRA AS A POTENTIAL ANTIMETASTATIC AGENT IN COLORECTAL ADENOCARCINOMA
}

\author{
${ }^{2}$ Robert Edwards, ${ }^{1}$ Kambiz Afrasiabi and ${ }^{1}$ Kehui Wang \\ ${ }^{1}$ Department of Medicine, Division of Hematology/Oncology, University of California, Irvine \\ ${ }^{2}$ Department of Pathology, University of California, Irvine
}

Received 2014-03-07; Revised 2014-03-15; Accepted 2014-03-26

\begin{abstract}
The family of Retinoic acid compounds have long been the subject of intense study and interest as chemo prophylactic agents. This dates back to their therapeutic effect in leukoplakia Where ATRA was shown to lead to the reversal of premalignant lesions and Subsequent reports on their use in metastatic colorectal adenocarcinoma and a vast array of other malignant disorders as well as adjuvant treatment of loco regional head and neck and colorectal adenocarcinoma. All-trans retinoic acid has transformed the treatment and outcome of patients with acute promyelocytic leukemia by differentiating leukemic promyelocytes into mature cells. Retinoid Receptor knock out mouse models also serve as excellent proof for the critical role of retinoids in embryogenesis, specifically their vital role in heart and blood vessel development, CNS specification, fetal lung development and proper limb morphogenesis. Here we show the results of our first phase of in vitro studies of the interaction of ATRA with the Gut homing Receptor, CCR9. Hypoxia up regulates CCR9 expression in CCIC, while ATRA down regulates CCR9 expression in hypoxic CCIC. In contrast, HT29 cells express significantly less CCR9 which is up regulated by hypoxia and further enhanced by ATRA treatment. We conclude that CCIC (representing a colon cancer stem-like line) respond differently to ATRA treatment Vs. a less stem-like, more differentiated cancer cell line such as HT-29. These results support a potential clinical use of ATRA early in the natural history of CRC in order to abort or slow down the process of metastasis in colorectal adenocarcinoma which is the ultimate killer of such patients. They also signify the critical significance of the time window and intratumoral heterogeneity in the final outcome of our intervention with ATRA in the treatment of CRC.
\end{abstract}

Keywords: ATRA, Microenvironment, CCR9, Metastasis

\section{INTRODUCTION}

The relationship of retinoid signaling with other cellular sub-compartments is of essence for cellular homeostasis and it plays a critical role in orchestrating their smooth function (Quadro et al., 2003; Perlmann and Jansson, 1995; Niles, 2004).

Furthermore, it is widely known that many stem cell compartments are characterized and regulated by their apoptotic machinery (Gudas and Wagner, 2011). By up regulating the BCLXL/BAX ratio in cells, retinoids are able to inhibit apoptosis in no transformed cells, while in SV40-transformed mammary cancer cells they promote apoptosis by altering this protein expression ratio.

The coordinated interplay between retinoids and metabolic pathways have thus allowed transit amplifying cells that are unable to proceed with differentiation to undergo apoptosis under normal physiological conditions (Noy, 2010; Sumantran et al., 2000; Rivenzon-Segal et al., 2000).

In addition any process that perturbs the fine balance of cell energetic in favor of an inappropriate decrease in the free energy of the cell would essentially freeze the

Corresponding Author: Kambiz Afrasiabi, Department of Medicine, Division of Hematology/Oncology, University of California, Irvine 
differentiating process and trigger activation of the apoptotic machinery which ultimately becomes defective in malignant cells (Rivenzon-Segal et al., 2000).

Retinoid activated channels modulating cGMP/GTP ratio alter cellular metabolism in such a way that the amount of free energy, specifically GTP and ATP available to the cell would remain above a critical threshold all the time (Kholodenko et al., 2007; Dey, 2007; Flanagan and Meckling-Gill, 1997). This mechanism also breaks down in malignant cell in which the de-differentiated cells with low free energy do not die, but rather divide rapidly (Kambiz-Afrasiabi, 2013).

\subsection{Warburg and Cancer Metabolism}

The source of energy and building blocks for these rapid divisions are provided by glycolytic pathway as well as glycine metabolism which supplies the purine and pyrimidine molecules needed for nucleic acid synthesis (Moreno-Sa et al., 2007).

Despite the fact that Warburg effect was first described several decades ago, there has been no convincing explanation thus far as to why Krebs cycle and electron transport system are not the primary energy suppliers for cancer cell metabolism.

Hypotheses about This explanation seem to be multifaceted. One hypothesis is that expansion of the hypoxic niche is necessary in maintaining the integrity of the cancer stem cell compartment, which automatically forbids oxidative phosphorylation (Soeda et al., 2009).

However there is a much deeper and more ancient mechanism favoring Warburg effect and Glycine metabolism in cancer cells, namely the fact that the birth of malignant phenotype coincides with the breakdown of the energetic homeostasis fabric, which has taken the cell into an irreversible low free energy state (Brahimi-Horn and Pouysségur, 2011).

A normal cell has mastered to keep the free energy at a maximum level as per the limits of the second law of thermodynamics beyond any other known and studied system (Bonnet et al., 2007; Kambiz-Afrasiabi, 2013).

The intracellular communication network which according to one measurement involves three hundred thousand protein-protein interactions is fine tuned to this simple and elegant principle. Because the malignant cell resides at a significantly lower free energy level which could potentially modify the quaternary structure of the protein components of the hairball and the real nature of their interactions. One example is stabilization of $\mathrm{HuR}$ by Beta-Catenin following translocation of Beta-Catenin from the plasma membrane to the cytoplasm under energy restrictive condition (D'Uva et al., 2013). Thus it is not surprising that these malignant protein-protein interactions are energetically successful with significantly less available free energy (West et al., 2012).

Retinoids should be considered the guardian of cell energetics because they make a paradoxical shift in their quaternary structure from low to high free energy/more stable isoform (from 11-cis to all trans retinoic acid) upon exposure to the damaging and destabilizing radiation, in contradistinction to other vital macromolecules such as DNA and RNA (Stecher et al., 1999; Villarroya et al., 2004).

It is easy to imagine that the incessant exposure of the normal cell to noxious environmental agents and radiation, creates a relentless challenge to the cell to maintain its normal energetics and the only evolved machinery that could acquire more stability and survival advantage under these conditions, namely Retinoids could take the lead to restore the energetic homeostasis and this has been an unappreciated concept of the critical role of Retinoid family (Reynolds, 2007; Herczenik and Gebbink, 2008).

The breakdown of energetics homeostasis and exceptional advantage of malignant cell to survive and thrive under low energy supply conditions and the dominance of glycolytic pathway and involvement of Glycine for purine and pyrimidine metabolism, as well as dysfunction of oxidative phosphorylation and electron transport system opens the way for expansion of the hypoxic niche (D'Uva et al., 2013; Lefebvre et al., 2006).

Such expansion is associated with the development of stemness capability of the cells that reside in the hypoxic niche. This translates into expansion of malignant stem cell compartment and further acquisitions of more malignant and aggressive mutations (Niederreither and Dollé, 2008; Krieg and Boyman, 2009).

\section{MATERIALS AND METHODS}

\subsection{CCIC Cells}

Colon cancer initiating cells were originally derived from a sporadic colon cancer patient's surgical sample at UCI in July 2007. These cells were injected subcutaneously into a nude mouse. We were able to replicate the original tumor phenotype in serial injections 
down to 100 cells, at which stage we called them CCIC. We put some aliquots in liquid nitrogen and continued to grow the rest in conventional stem cell culture medium. We noticed spheroid formation immediately.

\subsection{HT29 Cells}

These cells are commercially available sporadic MSS colon cancer cell lines. They formed spheroids in conventional stem cell medium. Spheroid formation was more brisk and effective in conditioned stem cell medium.

\subsection{ATRA}

All trans Retinoic acid was obtained commercially and a $30 \mathrm{mg}$ aliquot was dissolved in $20 \mathrm{cc} 100 \%$ ethyl alcohol $=30 \mathrm{mmol} /$ liter. $1 \mathrm{micromol} /$ liter dilution was added to 10 cc ultra low attachment flasks containing the spheroids.

\subsection{Hypoxic Chamber}

Conventional hypoxic chamber at $1 \% \mathrm{O} 2$ for $24-48 \mathrm{~h}$ was used all the time.

\subsection{Western Blot}

Using standard SDS gel, running and transfer buffer, western blot was done. 180 volts for 60-90 min was used for running and 30 volts overnight was used for transfer.

\subsection{Normalization}

Using conventional software programs, normalization of CCR9 and CCR9/Actin was performed.

\subsection{CCR9 Monoclonal Rabbit Antibody}

Commercially available Primary Ab, conc of $1 / 300$ and secondary Rabbit $1 / 3000$ with exposure time of 10 sec were used.

\subsection{Spheroid Formation}

In conventional stem cell medium including EGF, FGF and N2, HT29 cells formed well-defined spheroids.

\section{RESULTS}

CCR9 baseline expression in CCIC which represents colon cancer stem cell population is three times higher than HT29 and even though hypoxic microenvironment almost doubles that, ATRA has an insignificant effect on CCIC in this regard (Fig. 1). In contradistinction to CCIC, ATRA and hypoxia have more or less similar effect on over expression of CCR9 in HT29 cell line and there is close to a synergestic effect of the combination of these two on HT29 CCR9 expression (Fig. 2).

This signifies that CCIC that we have been growing for 5 years in culture medium (2007-2012) which could well have piled up mutations similar to metastatic colon adenocarcinoma stem cells over a time span of 5 years, do not significantly home back in response to ATRA, in contradistinctions to HT29 which represents sporadic colon cancer cell line early in its natural history. The same CCIC that we just got out of liquid nitrogen in 2012, tagged with W (Fig. 3), which represent colon cancer stem cells early in their natural history, act in an opposite way, i.e.,: ATRA would significantly reduce their homing receptor.

All these point towards the critical significance of the time interval in natural history of colon adenocarcinoma that we intervene with our anti metastatic agents. In case of CCIC even though their baseline CCR9 level is more than HT29 plus ATRA (Fig. 4), other variables such as CCL25 level and receptor sensitivity could be totally different in these aged colon cancer stem cell lines thus leading to their aggressive biological behavior and metastatic potential. these results indicate that a much deeper understanding of the nurturing gut and metastatic site Microenvironment and their related homing receptors and signaling pathways and their change throughout the natural history of these malignancies would lead to the design of much more refined treatment regimens in near future the outcome of metastatic.

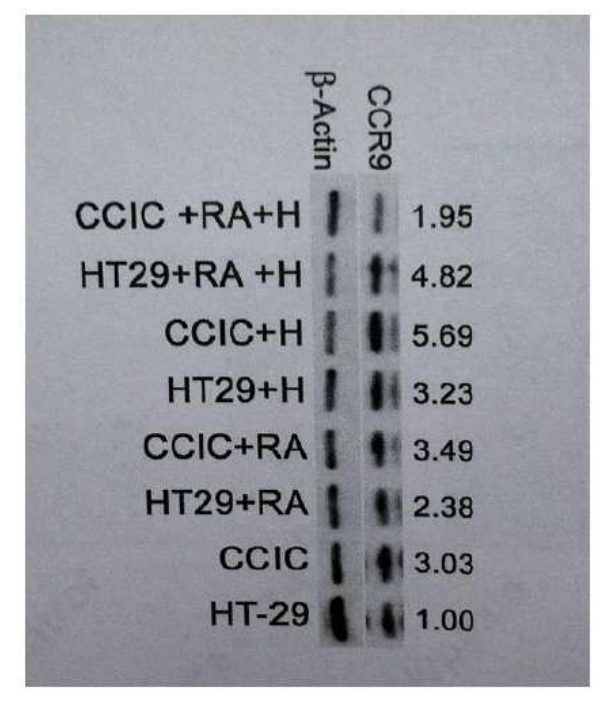

Fig. 1. Baseline expression of CCR9 and its modification with ATRA+/-hypoxia in CCIC and HT29 cell line 
Robert Edwards et al. / OnLine Journal of Biological Sciences 14 (2): 102-107, 2014

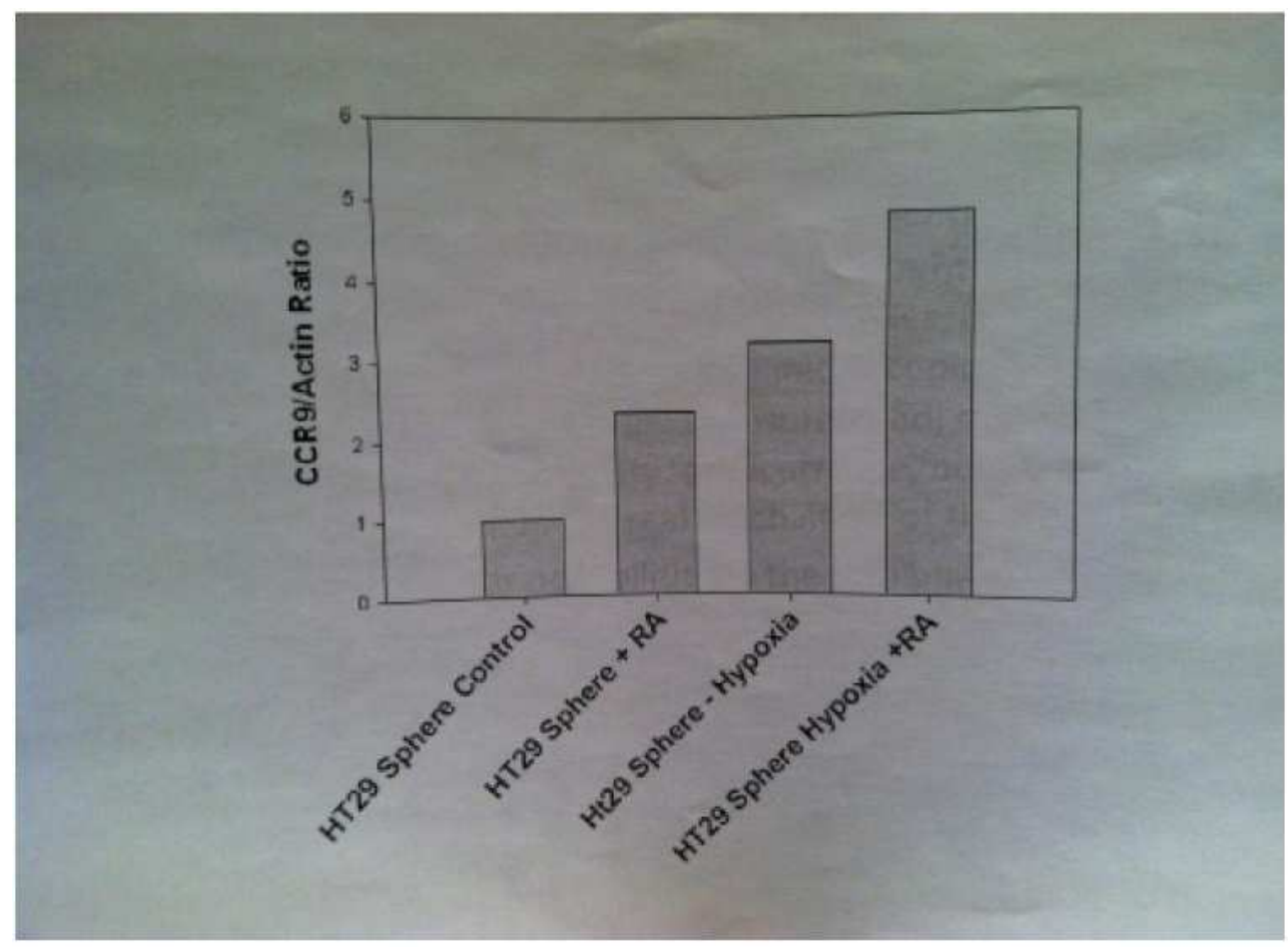

Fig. 2. Normalization of CCR9/ ACTIN clearly shows close to a synergistic effect of ATRA and hypoxia on CCR9 expression in HT29 cell line

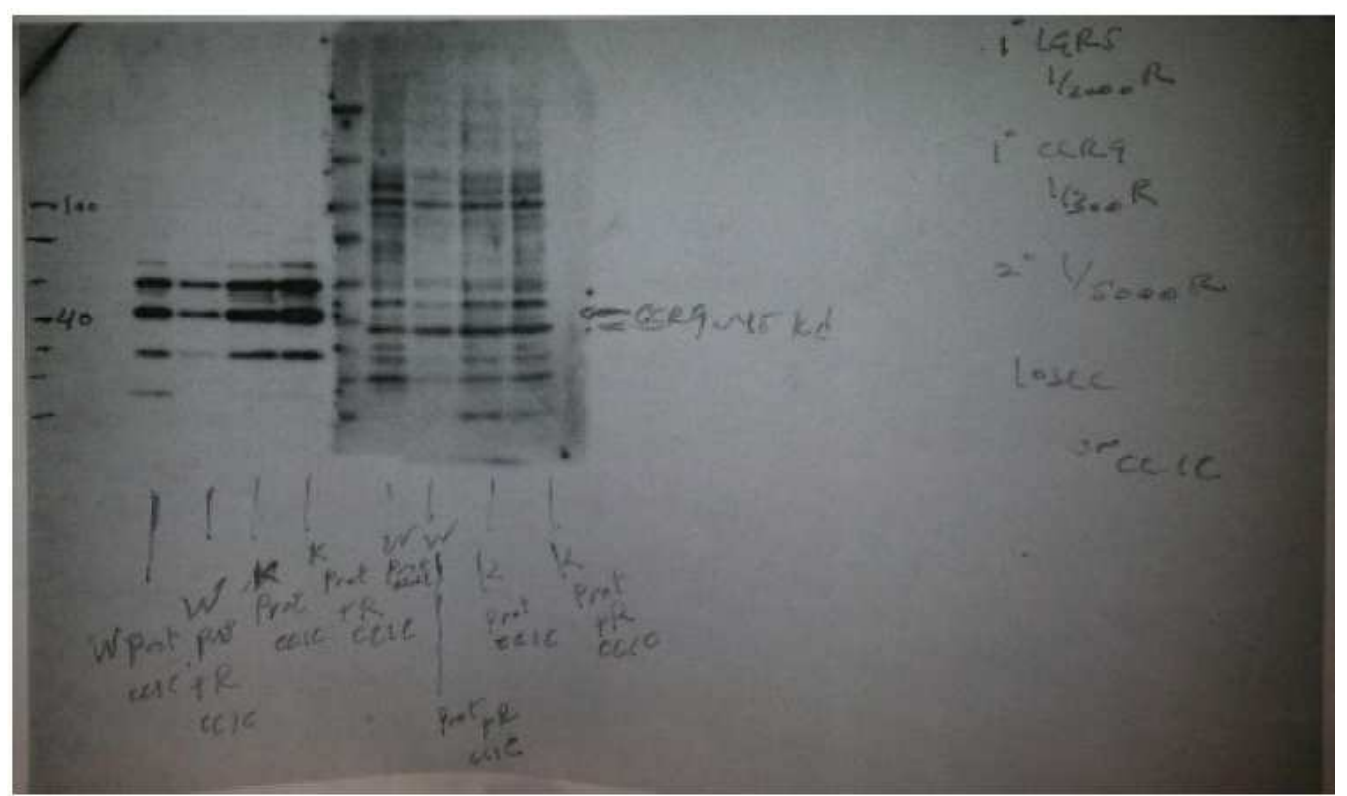

Fig. 3. Left and Right lanes tagged with W( fresh CCIC out of liquid nitrogen) and K( old CCIC in culture for 5 years). ATRA down regulates CCR9 in WCCIC and up regulates it in KCCIC 


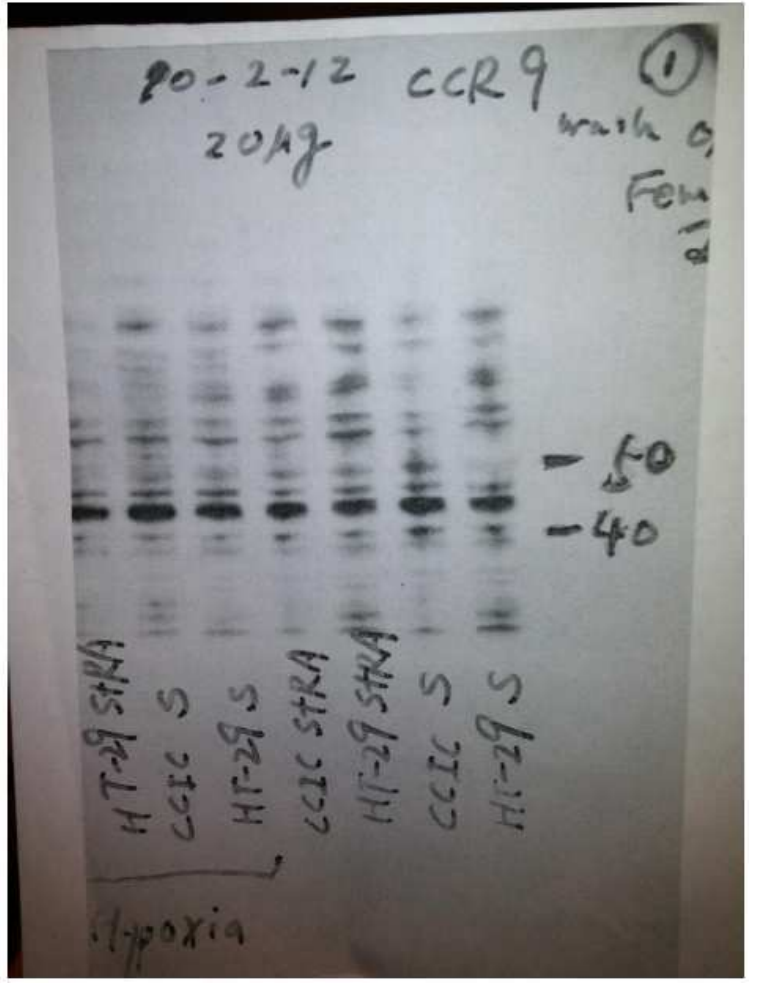

Fig. 4. CCR9 expression in hypoxia and normoxia, not normalized for actin in CCIc and HT29 cell line under hypoxic and normoxic conditions

\section{DISCUSSION}

The great majority of patients dying of colorectal adenocarcinoma die as a result of metastatic disease. We are in need of a new and radical approach to this problem on an urgent basis. Our curent treatment strategies in clinic have failed to make a meaningful achievement.

The preliminary results of our studies with ATRA suggest that there is an exciting opportunity available to us that we have not tried as yet.

These results also strongly suggest that time is of essence in strategezing our approach in this regard as far as the natural history of this disease is concerned.

Indeed we could get opposite results using the same agent at different time intervals following the original diagnosis.

These data also suggest that earlier intervention in natural history of colorectal adenocarcinoma could lead to more successful outcome and with less difficulty.

Another corrolary of these data is the birth of the new chapter of pre emptive therapy with Atra in colorectal adenocarcinoma.
Down regulation of CCR9 with ATRA in these patients could deprive them of the nurturing colorectal microenvironment which would allow them to develop the more malignant and aggressive mutations through time. By generation of this vulnerability in cancer cells and during this narrow time window and by using a combination of targetted and cytotoxic agents we could eradicate the clone that could lead to the future demise of the host. Furthermore by deriving the genetic and micro RNA signature of these circulating cancer cells we could further refine our treatment strategy.

In metastatic disease the thinking and straegy should be totally different. Here it is already too late to implement pre emptive treatmnt strategy. Rather we should learn more about the nurturing micro environment of metastatic sites as well as CCL25 dynamics and receptor sensitivity and focus our efforts on those issues. This time by interfering with the homing of colorectal cancer cells in foreign organs and taking them out of their nurturing metastatic microenvironment and by using cytotoxic and targetted therapy we have a significantly higher chance of coming up with meaningful results.

In closing any future treatment strategy for colorectal adenocarcinoma should put interference with the supportive microenvironment as its centerpiece. We should also remain cognizant of the significance of intratumoral heterogeneity and interpatient differences in Gene and Micro RNA signatures. All these should act as the template for our future customized colorectal cancer treatment strategies. The era of one size fits all thinking and treatment should soon become history. These results could also well compare with manipulation of microenvironment with agents such as IMIDS in diseases such as multiple myeloma and MDS, which Without doubt have dramatically improved the outcome of those devastating disorders.

\section{CONCLUSION}

Our findings have opened the door on a new approach to treatment of colorectal adenocarcinoma both in early stage and metastatic disease, based on dissection of the dynamics of the loco regional and metastatic site microenvironment and interference with their feeding loops including CCR9 and its ligand in a time sensitive fashion. 


\section{REFERENCES}

Bonnet, S., S.L. Archer, J. Allalunis-Turner, A. Haromy and C. Beaulieu et al., 2007. A mitochondria-K+ channel axis is suppressed in cancer and its normalization promotes apoptosis and inhibits cancer growth. Cancer Cell, 11: 37-51. PMID: 17222789

Brahimi-Horn, M.C. and B.G.J. Pouysségur, 2011. Hypoxia and energetic tumour metabolism. Curr. Opin. Genet., 21: 67-72. DOI: 10.1016/j.gde.2010.10.006

Dey, N., 2007. Molecular and cellular biology: CSK controls RAR signaling. MCB.

Flanagan, S.A. and K.A. Meckling-Gill, 1997. Characterization of a novel nat-dependent, guanosine-specific, nitrobenzylthioinosine-sensitive transporter in acute promyelocytic leukemia cells. J. Biol. Chem., 272: 18026-32. PMID: 9218431

D’Uva, G., S. Bertoni, M. Lauriola, S. De Carolis and A. Pacilli et al., 2013. Beta-Catenin/HuR posttranscriptional machinery governs cancer stem cell features in response to hypoxia. PLoS One, 8: e80742-e80742.

Gudas, L.J. and J.A. Wagner, 2011. Retinoids regulate stem cell differentiation. J. Cell Physiol., 226: 32230. DOI: $10.1002 /$ jcp. 22417

Herczenik, E. and M.F. Gebbink, 2008. Molecular and cellular aspects of protein misfolding and disease. Eszter Herczenik. FASEB J., 22: 2115-33. DOI: 10.1096/fj.07-099671

Kambiz-Afrasiabi, 2013. Free energy measurement distinguishes cancer cell from normal cell. Online J. Biol. Sci., 13: 121-125. DOI: 10.3844/ojbsci.2013.121.125

Kholodenko, R., I. Kholodenko, V. Sorokin, A. Tolmazova and O. Sazonova et al., 2007. Anti-apoptotic effect of retinoic acid on retinal progenitor cells mediated by a protein kinase A-dependent mechanism. Cell Res., 17: 151-62. DOI:10.1038/sj.cr.7310147

Krieg, C. and O. Boyman, 2009. The role of chemokines in cancer immune surveillance by the adaptive immune system. Semin Cancer Biol., 19: 76-83. PMID: 19038343

Lefebvre, P., G. Chinetti, J.C. Fruchart and B. Staels, 2006. Sorting the role of PPARa in energy metabolism and vascular homeostasis. Jclin Invest, 116: 571-580. DOI: 10.1172/jci27989

Moreno-Sa, R., S. Nchez, R. Enrıquez, A. Marın-Herna and E. Saavedra, 2007. Energy metabolism in tumor cells. Rafael Moreno-Sanchez FEBS J., 274: 13931418. PMID: 17302740
Niederreither, K. and P. Dollé, 2008. Retinoic acid in development: towards an integrated view. Nat. Rev. Genetics, 9: 541-553. PMID: 18542081

Niles, R.M., 2004. Signaling pathways in retinoid chemoprevention and treatment of cancer. Mutat. Res., 555: 81-96. DOI: 10.1016/j.mrfmmm.2004.05.020

Noy, N., 2010. Between death and survival: Retinoic acid in regulation of apoptosis. Annu. Rev. Nutr., 21: 201-17. DOI: 10.1146/annurev.nutr.28.061807.155509

Perlmann, T. and L. Jansson, 1995. A novel pathway for vitamin A signaling mediated by RXR heterodimerization with NGFI-B and NURR1. Genes Dev., 1: 769-82. PMID: 7705655

Quadro, L., L. Hamberger, V. Colantuoni, M.E. Gottesman and W.S. Blaner, 2003. Understanding the physiological role of retinol-binding protein in vitamin a metabolism using transgenic and knockout mouse models. Mol. Aspects Med., 24: 421-30. DOI: 10.1016/S0098-2997(03)00038-4

Reynolds, C.P., 2007. Differentiating agents in pediatric malignancies: Retinoids in neuroblastoma. Curr. Oncol. Rep., 2: 511-8. PMID: 11122886

Rivenzon-Segal, D., E. Rushkin, S. Polak-Charcon and H. Degani, 2000. Glucose transporters and transport kinetics in retinoic acid-differentiated T47D human breast cancer cells. Am. J. Physiol. Endocrinol. Metabolism, 279: E508-E519. PMID: 10950817

Soeda, A., M. Park, D. Lee, A. Mintz and A. Androutsellis-Theotokis et al., 2009. Hypoxia promotes expansion of the CD133-positive glioma stem cells through activation of HIF-1alpha. Oncogene, 45: 3949-59. DOI: 10.1038/onc.2009.252

Stecher, H., M.H. Gelb, J.C. Saari and K. Palczewski, 1999. Preferential release of 11-cis Retinol from retinal pigment epithelial cells in the presence of Retinaldehyde binding protein. J. Biol. Chem., 274: 8577-85. PMID: 10085092

Sumantran, V.N., R. Zhang, D.S. Lee and M.S. Wicha, 2000. Differential regulation of apoptosis in normal versus transformed mammary epithelium by lutein and retinoic acid. Cancer Epidemiol. Biomarkers Prev., 9: 257-63. PMID: 10750663

Villarroya, F., R. Iglesias and M. Giralt, 2004. Retinoids and retinoid receptors in the control of energy balance: Novel pharmacological strategies in obesity and diabetes Curr. Med. Chem., 11: 795-805. PMID: 15032732

West, J., G. Bianconi, S. Severini and A.E. Teschendorff, 2012. Differential network entropy reveals cancer system hallmarks. Scientif. Reports, 2: 802-802. PMID: 23150773 